\section{Tendência da mortalidade por doenças neoplásicas em 10 capitais brasileiras, de 1980 a 2000}

\section{Cancer mortality trends in 10 Brazilian capitals, 1980 - 2000}

\section{Adriane Cervi' ${ }^{1}$}

\section{Helen Hermana Miranda Hermsdorff ${ }^{2}$}

\section{Rita de Cássia Lanes Ribeiro ${ }^{3}$}

${ }^{1}$ Programa de Pós-Graduação em Ciência da Nutrição, Departamento de Nutrição e Saúde, Universidade Federal de Viçosa - UFV,Viçosa/MG; Departamento de Ciências da Saúde, Universidade Regional do Noroeste do Estado do Rio Grande do Sul - UNIJUÍ, ljuí/RS

${ }^{2}$ Programa de Pós-Graduação em Ciência da Nutrição, Departamento de Nutrição e Saúde, Universidade Federal de Viçosa.

${ }^{3}$ Departamento de Nutrição e Saúde, Universidade Federal de Viçosa

\section{Resumo}

O objetivo deste estudo foi analisar a tendência da mortalidade por doenças neoplásicas, segundo sexo e faixa etária, no período de 1980 a 2000, em 10 capitais brasileiras. Para tanto, realizou-se um estudo ecológico de série temporal, utilizando dados de óbitos de indivíduos acima de 30 anos de ambos os sexos. Os dados de mortalidade foram obtidos do Sistema de Informação sobre Mortalidade do Ministério da Saúde (SIM/MS/ DATASUS). Utilizou-se a padronização dos coeficientes de mortalidade pelo método direto e o método de regressão linear simples. O coeficiente médio padronizado de mortalidade por neoplasia, estimado entre 1980 e 2000, foi de 10.239 mortes, com aumento de 5.220 mortes por 100 mil habitantes ao ano. Os coeficientes de mortalidade foram maiores no sexo masculino e indivíduos acima de 60 anos. Observou-se tendência crescente da mortalidade nas capitais analisadas, em ambos os grupos etários, sendo maior nas capitais das regiões Centro-Oeste, Norte e Nordeste e na população acima de 60 anos. A elevada mortalidade para o sexo masculino e a população idosa deve-se, provavelmente, à maior exposição aos fatores de risco ambientais e à maior prevalência de cânceres letais entre os homens. As capitais das regiões Sul e Sudeste seguem tendência crescente, porém com incremento menor comparado às demais, provavelmente devido aos maiores avanços no processo de transição demográfica e epidemiológica, e melhores serviços de assistência médica. Este estudo mostrou que a mortalidade por neoplasia aumentou ao longo da série temporal, podendo se tornar a principal causa de morte no Brasil.

Palavras-chave: Estudo Ecológico. Mortalidade. Doenças Neoplásicas. Câncer 
Abstract

The objective of this study was to analyze the cancer mortality trends, according to gender and age, in the period from 1980 to 2000 , in ten Brazilian capitals. To that end, an ecological time series study was carried out, using mortality data of subjects above 30 years of age and of both genders. Mortality data were obtained from the Mortality Information System of the Ministry of Health (SIM/MS/DATASUS). Mortality rates were standardized by the direct method and the simple linear regression method. The average standardized mortality rate for cancer, estimated between 1980 and 2000, was 10,239 deaths, with an increase of 5,220 deaths per 100,000 inhabitants for each year. Mortality rates were higher in men and individuals above 60 years of age. A growing trend in mortality was observed in the capitals analyzed, in both age groups, and was higher in the capitals of the North, Northwest and Center-West regions and in the population above 60 years of age. The high mortality for men and the elderly population was probably due to the higher exposure to environmental risk factors and greater prevalence of lethal cancers among men. The capitals of the South and Southeast regions followed the growing trend, with a smaller increase in comparison to the others, due to the largest advances in the demographic and epidemiologic transition process and better health care. This study showed that the mortality due to cancer has increased in the time series, and may possibly become the major cause of death in Brazil.

Keywords: Ecological Study. Mortality. Neoplasic disease. Cancer.

\section{Introdução}

O Brasil e diversos países da América Latina estão experimentando, nos últimos 20 anos, uma rápida transição demográfica, epidemiológica e nutricional ${ }^{1}$. A mudança na estrutura etária da população brasileira iniciou-se na década de 40 , mas esta tendência acentuou-se nos anos 70, quando a participação dos indivíduos com 60 anos ou mais passou de $5 \%$ para $6,1 \%$ em 1980, chegando a 7,7\% em $1991^{2-3}$. Em 2000, do total de habitantes, 15,5 milhões possuíam 60 anos ou mais, representando cerca de $10 \%$ da população geral ${ }^{4}$.

Associada ao envelhecimento acelerado da população, o processo de urbanização e as mudanças socioeconômicas, alimentares e no estilo de vida, houve uma conseqüente modificação no perfil de morbi-mortalidade nos últimos 20 anos, caracterizando a transição epidemiológica no Brasil ${ }^{5}$. Dentre as modificações mais relevantes destacam-se: (1) a melhoria nas condições de saneamento e moradia; (2) o acesso a serviços de atenção à saúde, incluindo o avanço de sua tecnologia; (3) a queda da taxa de fecundidade; (4) a maior exposição a determinados riscos ambientais; (5) a preferência por alimentos refinados, ricos em gorduras, em detrimento ao consumo de frutas e hortaliças; (6) o aumento do sedentarismo ${ }^{6-8}$.

Desta forma, verificou-se uma redução das gastroenterites, sarampo e outras enfermidades incidentes na infância, concomitante a um aumento na prevalência e incidência de doenças crônico-degenerativas ou não transmissíveis relacionadas à obesidade, como diabetes tipo 2, doenças cardiovasculares, hipertensão arterial e diversos tipos de câncer ${ }^{9}$.

As alterações no padrão alimentar têm importante papel na determinação do risco de neoplasias. Segundo Sichieri et al. ${ }^{10}$, a ingestão energética excessiva está associada positivamente ao aumento do risco de câncer de cólon, pulmão e esôfago, e o aumento do consumo de alimentos ricos em gordura ao aumento do risco de cân- 
cer de cólon. A redução no consumo de frutas, hortaliças (fontes de fibras), concomitante ou não ao aumento do consumo de alimentos ricos em gorduras, está correlacionada ao maior risco de câncer de estômago, cólon e reto ${ }^{10-11}$.

Analisando-se a mortalidade do Brasil em 1998, os óbitos por neoplasias estão entre as principais causas nas diversas regiões do país, ao lado das doenças do aparelho circulatório (32,6\%), causas externas $(14,6 \%)$, doenças do aparelho respiratório $(11,6 \%)$, afecções do período perinatal $(4,6 \%)$ e doenças infecciosas e parasitárias $(6,2 \%)^{12}$. Em 1997, a proporção de mortalidade por neoplasias em relação ao total de mortes por região distribuía-se na seguinte ordem: 8,21\% Nordeste; 9,11\% Norte; $12,3 \%$ Centro Oeste; $13,96 \%$ Sudeste; $17,07 \%$ Sul $^{13}$. De acordo com Faria et al. ${ }^{14}$, os maiores valores apresentados nas regiões Sudeste e Sul podem indicar a importância do processo de urbanização e industrialização na transição epidemiológica. Atualmente, o câncer constitui a segunda causa de morte por doença no Brasil, e estudos de tendência sugerem que posteriormente poderá superar a mortalidade por doenças cardiovasculares, tornando-se a principal causa de morte no país ${ }^{15}$. Em 2000, as neoplasias foram responsáveis por $12,73 \%$ dos 946.392 óbitos registrados no Brasil, sendo que 53,97\% dos óbitos por neoplasia ocorreram entre os homens e $46,01 \%$ entre as mulheres $^{13}$. Em relação aos principais tipos de cânceres, destaca-se pulmão (12,4\%), estômago $(9,8 \%)$, mama $(7,3 \%)$, próstata $(6,5 \%)$, cólon e reto $(6,2 \%)$ e colo e útero $(5,8 \%)^{12}$.

As altas prevalências e incidências de neoplasia, bem como a alta letalidade das mesmas, são causa de grande interesse de pesquisadores em estudos epidemiológicos. Trabalhos que avaliam os coeficientes de morbi-mortalidade, relacionados às doenças neoplásicas como um todo, são importantes para o monitoramento da mortalidade no Brasil, permitindo caracterizar populações de risco nas diversas regiões, de modo a otimizar a definição de políticas de saúde pública que visem a prevenção, proteção e atenção à saúde.

Desse modo, o objetivo do presente estudo foi analisar a tendência da mortalidade por doenças neoplásicas, segundo sexo e faixa etária, no período de 1980 a 2000 em 10 capitais brasileiras, relacionando os dados obtidos com o processo de transição demográfica, nutricional e epidemiológica no Brasil.

\section{Metodologia}

Realizou-se um estudo ecológico de série temporal, utilizando dados de óbitos de indivíduos com idade acima de 30 anos, de ambos os sexos, no período de 1980 a 2000 em 10 capitais do país. Foram incluídas capitais específicas, principais metrópoles de cada macro-região do Brasil, a saber: Curitiba e Porto Alegre (Sul); Belo Horizonte, Rio de Janeiro e São Paulo (Sudeste); Manaus (Norte); Salvador e Recife (Nordeste); Cuiabá e Distrito Federal (Centro-Oeste).

As estimativas da população por sexo e faixa etária (30-59 e > 60 anos) foram obtidas do Instituto Brasileiro de Geografia e Estatística (IBGE), calculadas para $1^{\circ}$ de julho de cada ano, sendo utilizadas para o cálculo dos coeficientes de mortalidade para neoplasia.

Os dados de mortalidade foram obtidos diretamente do Sistema de Informação sobre Mortalidade (SIM) do Ministério da Saúde (SIM/MS/DATASUS). Calculou-se o coeficiente de mortalidade por neoplasia segundo sexo, grupos etários e capitais em cada ano investigado, analisando-se os óbitos segundo a Classificação Internacional de Doenças (CID): Neoplasia (tumores) (capítulos II da CID-9 e da CID-10).

Realizou-se a padronização dos coeficientes de mortalidade pelo método direto, considerando a população brasileira de 1991 como população padrão ${ }^{15-16}$. Os coeficientes foram calculados para cada ano do período estudado e para cada capital, utilizando-se tanto o número total de óbitos, como os dados separados para cada sexo.

Para a análise de tendências temporais de mortalidade foram utilizados os coefici- 
entes de mortalidade padronizados e o método de regressão linear simples. Neste método, os coeficientes de mortalidade padronizados foram considerados como variável dependente $(\mathrm{Y})$, e os anos calendário de estudo como variável independente (X). Transformou-se a variável ano na variável centralizada (ano - 1990), sendo este o ponto médio da série histórica. Dessa maneira, obteve-se o modelo estimado: $\mathrm{Y}=\mathrm{b}_{0}$ $+\mathrm{b}_{1} \mathrm{X}$ onde $\mathrm{Y}=$ coeficiente padronizado, $\mathrm{b}_{0}=$ coeficiente médio do período, $b_{1}=$ incremento anual médio e $\mathrm{X}=$ ano-1990.

\section{Resultados}

Os homens apresentaram maiores coeficientes de mortalidade por neoplasia quando comparados às mulheres, havendo, para ambos os sexos, uma tendência de aumen- to ao longo da série temporal (1980-2000), intercalados com alguns períodos de redução. Em 1980, os coeficientes de mortalidade para o sexo masculino e feminino eram, respectivamente, 117,53 e 90,67 óbitos por 100 mil habitantes, 160,57 e 126,74 em 1990 e 235,77 e 187,45 em 2000 (Tabela 1).

Quando a população estudada foi estratificada por faixa etária e sexo, os maiores coeficientes foram encontrados nos indivíduos acima de 60 anos. No sexo masculino, houve um aumento de $60,3 \%$ na mortalidade entre a faixa etária de 30 a 59 anos, enquanto no feminino o aumento foi de $73,6 \%$. No grupo etário de 60 anos ou mais, os percentuais de incremento foram de $126,6 \%$ para homens e $133,0 \%$ para mulheres. Em geral, os percentuais de incremento da mortalidade por neoplasia foram de 17,09\% entre 1980 e 1985; 17,9\%

Tabela 1 - Coeficientes de mortalidade por neoplasia* (por 100.000 habitantes) segundo sexo e faixa etária, Brasil, 1980-2000.

Table 1 - Mortality Coefficients for cancer* (per 100,000 inhabitants) according to gender and age group, Brazil, 1980-2000.

\begin{tabular}{cccccccccc}
\hline $\begin{array}{c}\text { Ano } \\
\text { do }\end{array}$ & \multicolumn{3}{c}{ Masculino } & \multicolumn{3}{c}{ Feminino } \\
Óbito & $\begin{array}{c}\text { anos ou } \\
\text { + anos }\end{array}$ & Total & $\begin{array}{c}30-59 \\
\text { anos }\end{array}$ & $\begin{array}{c}60 \text { ou } \\
+ \text { anos }\end{array}$ & Total & $\begin{array}{c}30-59 \\
\text { anos }\end{array}$ & $\begin{array}{c}\text { Total ou } \\
+ \text { anos }\end{array}$ & Total \\
\hline 1980 & 56,75 & 381,33 & 117,53 & 50,45 & 246,87 & 90,67 & 53,52 & 308,71 & 103,62 \\
1981 & 58,44 & 394,25 & 121,32 & 51,66 & 252,33 & 92,75 & 54,96 & 317,60 & 106,53 \\
1982 & 59,88 & 409,09 & 125,27 & 51,59 & 266,00 & 95,50 & 55,63 & 331,81 & 109,85 \\
1983 & 61,93 & 433,34 & 131,48 & 53,32 & 277,75 & 99,28 & 57,52 & 349,31 & 114,81 \\
1984 & 61,94 & 449,69 & 134,55 & 55,21 & 283,06 & 101,87 & 58,49 & 359,69 & 117,63 \\
1985 & 63,50 & 463,44 & 138,39 & 56,39 & 295,98 & 105,45 & 59,86 & 372,99 & 121,33 \\
1986 & 65,00 & 480,10 & 142,73 & 57,70 & 314,92 & 110,37 & 61,26 & 390,89 & 125,97 \\
1987 & 67,21 & 499,43 & 148,14 & 59,78 & 326,80 & 114,46 & 63,40 & 406,19 & 130,70 \\
1988 & 68,00 & 527,88 & 154,11 & 61,74 & 341,89 & 119,11 & 64,79 & 427,43 & 135,98 \\
1989 & 67,75 & 540,07 & 156,19 & 62,44 & 354,62 & 122,27 & 65,03 & 439,91 & 138,63 \\
1990 & 69,04 & 557,87 & 160,57 & 64,23 & 369,49 & 126,74 & 66,58 & 456,13 & 143,06 \\
1991 & 68,59 & 588,71 & 165,98 & 65,0 & 386,58 & 131,41 & 67,11 & 479,54 & 148,08 \\
1992 & 72,58 & 621,91 & 175,44 & 68,45 & 409,27 & 138,24 & 70,46 & 507,06 & 156,18 \\
1993 & 75,04 & 641,90 & 181,19 & 69,89 & 420,67 & 141,73 & 72,40 & 522,41 & 160,75 \\
1994 & 77,15 & 660,62 & 186,40 & 73,25 & 440,80 & 148,51 & 75,15 & 541,90 & 166,78 \\
1995 & 77,29 & 686,76 & 191,41 & 73,82 & 460,33 & 152,97 & 75,51 & 564,47 & 171,51 \\
1996 & 80,63 & 725,69 & 201,42 & 76,91 & 479,34 & 159,32 & 78,72 & 592,64 & 179,62 \\
1997 & 82,50 & 757,31 & 208,86 & 79,45 & 496,47 & 164,85 & 80,94 & 616,43 & 186,07 \\
1998 & 86,05 & 783,24 & 216,60 & 83,20 & 509,96 & 170,59 & 84,59 & 635,64 & 192,78 \\
1999 & 89,49 & 820,15 & 226,31 & 86,03 & 542,37 & 179,48 & 87,72 & 670,12 & 202,06 \\
2000 & 90,98 & 864,23 & 235,77 & 87,59 & 575,21 & 187,45 & 89,24 & 708,13 & 210,75 \\
\hline
\end{tabular}

* Padronizado pela população brasileira de 1991.

* Standardized for the Brazilian population (1991). 
entre 1985 e 1990; 19,9\% entre 1990 e 1995 e 22,87\% entre 1995 e 2000 (Tabela 1).

A análise de tendência dos coeficientes de mortalidade padronizados pelo método de regressão linear simples mostrou que: a) o coeficiente padronizado médio de mortalidade estimado entre 1980 e 2000 foi de $10.239\left(\mathrm{R}^{2}=0,982 \mathrm{p}<0,001\right)$ mortes por 100 mil habitantes, com aumento de 5,220 mortes por 100 mil habitantes ao ano; b) o coeficiente padronizado médio para o período nos homens foi de $11.183\left(\mathrm{R}^{2}=0,980 \mathrm{p}<\right.$ $0,001)$ mortes por 100 mil habitantes, com aumento de 5,704 mortes por 100 mil habitantes; c) o coeficiente padronizado médio para o período nas mulheres foi de $9.359\left(\mathrm{R}^{2}\right.$ $=0,984 \mathrm{p}<0,001)$ mortes por $100 \mathrm{mil}$ habitantes, com aumento de 4,769 mortes por 100 mil habitantes (Figura 1).

Quando analisados os resultados por capitais, observaram-se diferenças entre os coeficientes encontrados no grupo etário de 30 a 59 anos e no grupo de 60 anos ou mais, bem como entre as regiões investigadas a partir de algumas capitais, mas sempre mostrando tendência crescente. Nas capi-
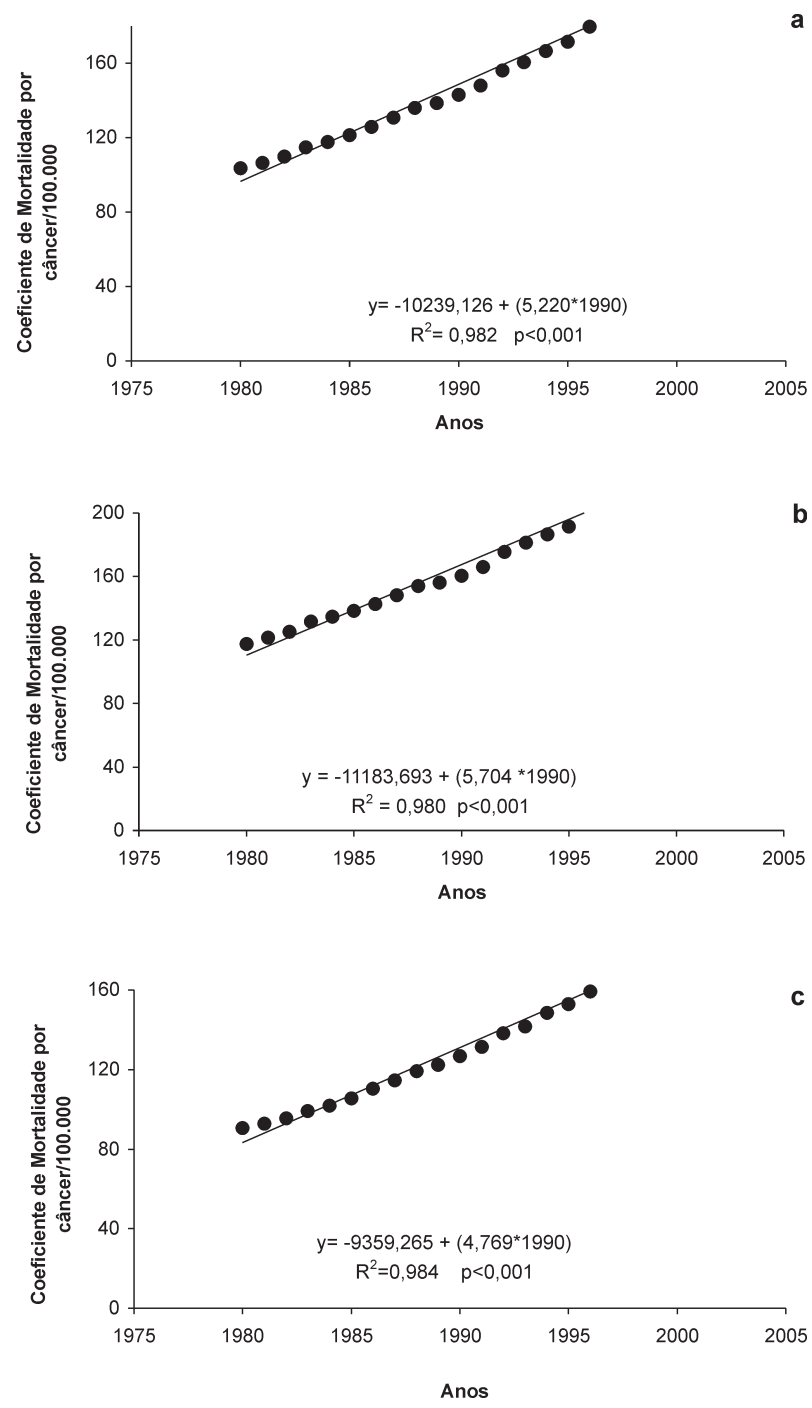

Figura 1 - Tendências dos coeficientes de mortalidade* por neoplasia, Brasil, 1980-2000. Ambos os sexos (a), homens (b) e mulheres (c).

Figure 1 -Trends of cancer mortality coefficients*. Brazil, 1980-2000. Both genders(a), men (b) and women (c).

* Padronizados pela população brasileira de 1991. * Standardized for the Brazilian population (1991). 
tais Curitiba, São Paulo, Belo Horizonte, Distrito Federal, Recife e Salvador, houve um aumento contínuo dos coeficientes de mortalidade por neoplasia, para a população entre 30 e 59 anos, no período de 1980 a 2000. Em Porto Alegre houve um leve declínio entre 1985 e 1995, enquanto na capital Rio de Janeiro houve uma redução entre 1990 a 1995 (Figura 2). Em Cuiabá, os coeficientes tiveram tendência crescente até 1995 e leve redução de 1995 a 2000, en- quanto Manaus teve leve redução no período entre 1985 a 1990 (Figura 3). Em relação à população com 60 anos ou mais, os coeficientes de mortalidade por neoplasia também apresentaram tendência crescente, no período de 1980 a 2000, na maioria das capitais investigadas, com exceção das capitais Recife, Manaus, Curitiba e Rio de Janeiro que tiveram tendência decrescente entre 1985 a 1990 e aumento contínuo a partir de então (Figuras 4 e 5).

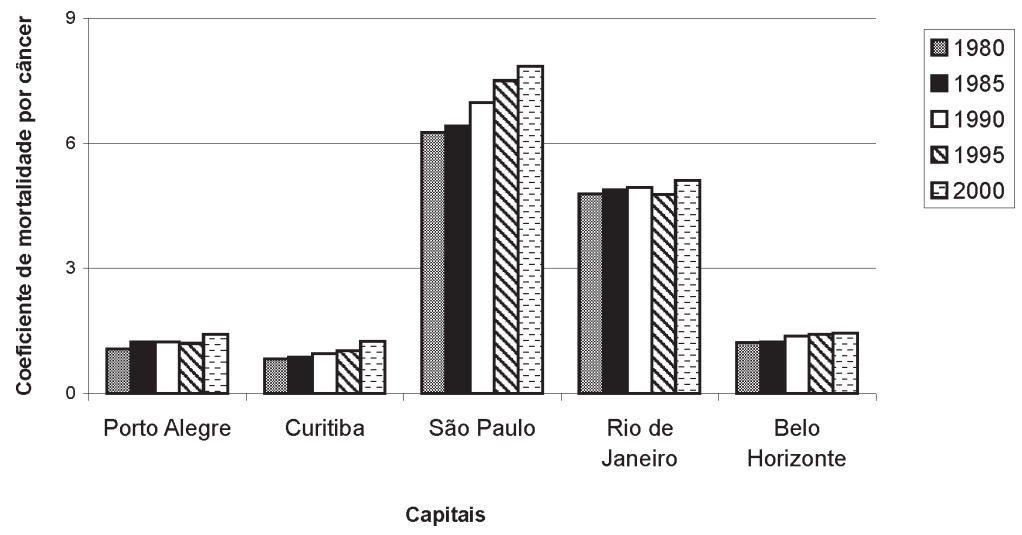

Figura 2 - Coeficientes de mortalidade por neoplasia* (por 100.000 habitantes) na população entre 30 a 59 anos, segundo ano e áreas metropolitanas das regiões Sul e Sudeste. Brasil, 19802000.

Figure 2 - Cancer mortality coefficients* (per 100,000 inhabitants), in the population between 30 and 59 years of age, according to year and metropolitan area of the South and Southeast regions. Brazil, 1980-2000.

* Padronizados pela população brasileira de 1991. * Standardized for the Brazilian population (1991).

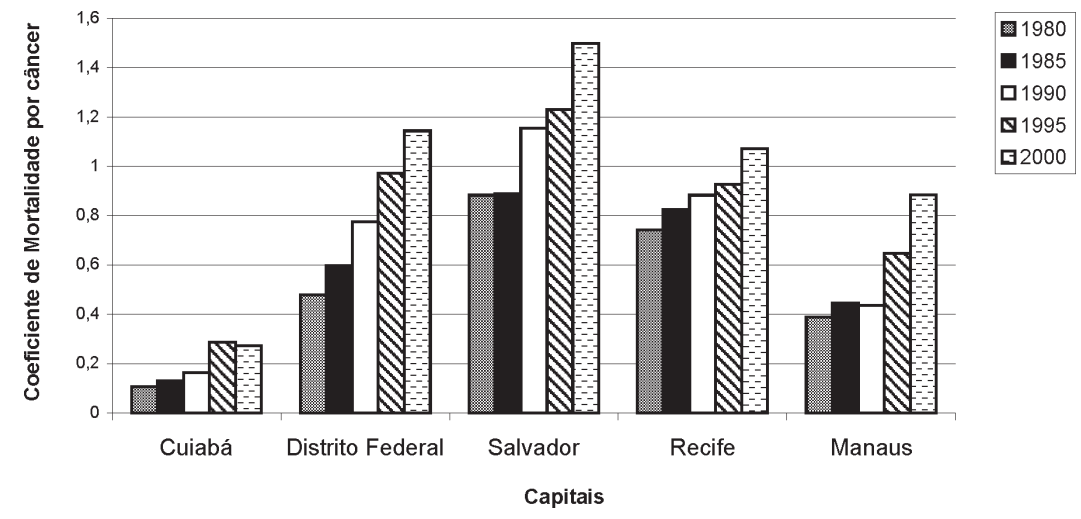

Figura 3- Coeficientes de mortalidade por neoplasia* (por 100.000 habitantes) na população entre 30 a 59 anos, segundo ano e áreas metropolitanas das regiões Norte, Nordeste e CentroOeste. Brasil, 1980-2000.

Figure 3 - Cancer mortality coefficients ${ }^{*}$ (per 100,000 inhabitants), in the population between 30 and 59 years of age, according to year and metropolitan area of the North, Northwest and Center-West regions. Brazil, 1980-2000.

* Padronizados pela população brasileira de 1991. * Standardized for the Brazilian population (1991). 


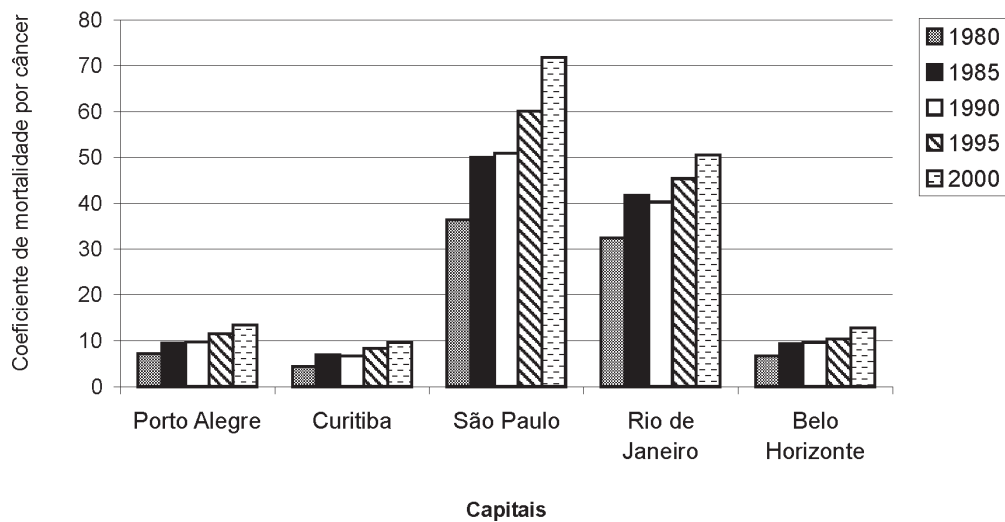

Figura 4- Coeficientes de mortalidade por neoplasia* (por 100.000 habitantes) na população acima de 60 anos, segundo ano e áreas metropolitanas das regiões Sul e Sudeste.Brasil, 19802000.

Figure 4- Cancer mortality coefficients" (per 100,000 inhabitants), in the population above 60 years of age, according to year and metropolitan area of the South and Southeast regions.

Brazil, 1980-2000.

* Padronizados pela população brasileira de 1991. * Standardized for the Brazilian population (1991).

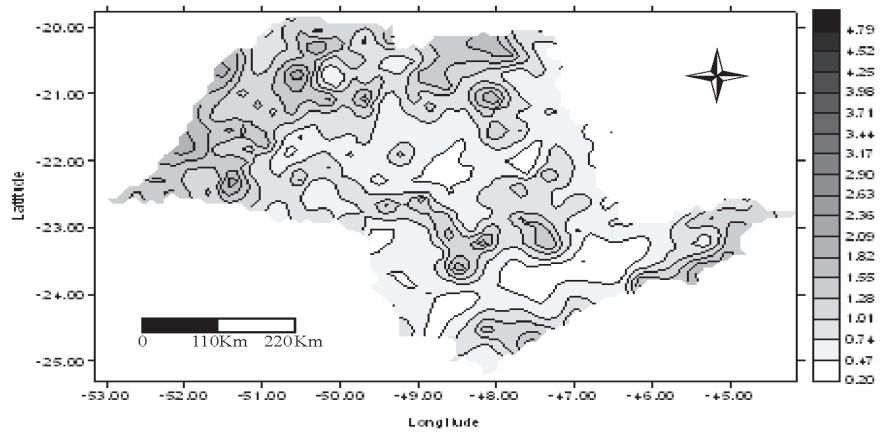

Figura 5 - Coeficientes de mortalidade por neoplasia* (por 100.000 habitantes) na população acima de 60 anos, segundo ano e áreas metropolitanas das regiões Norte, Nordeste e CentroOeste.Brasil, 1980-2000.

Figure 5 - Cancer mortality coefficients * (per 100,000 inhabitants), in the population above 60 years, according to year and metropolitan area of the North, Northwest and Center-West regions. Brazil, 1980-2000.

* Padronizados pela população brasileira de 1991. * Standardized for the Brazilian population (1991).

\section{Discussão}

Pelo método de regressão linear simples, foi observada uma tendência crescente da mortalidade por doenças neoplásicas ao longo dos 20 anos estudados, em ambos os sexos (Figura 1). Além disso, os coeficientes de mortalidade padronizados foram superiores para o sexo masculino e indivíduos acima de 60 anos (Tabela 1).

Os processos de transição demográfica, epidemiológica e nutricional experimenta- dos pelo Brasil nos últimos vinte anos ${ }^{1}$ poderiam explicar parcialmente os resultados apresentados acima. No final da década de 60 iniciou-se rápido e generalizado declínio da fecundidade no Brasil. Como conseqüência, a população brasileira entrou em contínuo processo de estreitamento da base da pirâmide demográfica, ou seja, ao envelhecimento da população ${ }^{3}$. O aumento crescente de indivíduos com mais de 60 anos tem levado ao incremento de doenças crônicas ligadas ao maior tempo de ex- 
posição aos fatores de risco, tais como, doenças cardiovasculares, obesidade, diabetes, hipertensão e câncer ${ }^{17-18}$.

Dentre os fatores determinantes da transição nutricional, e conseqüente aumento das doenças crônicas e degenerativas no Brasil e na América Latina, destacam-se o abandono de dietas ricas em fibras e grãos, frutas, verduras e legumes, bem como o aumento do consumo de açúcares e gorduras saturadas, aumento, portanto, da densidade calórica dos alimentos ingeridos ${ }^{8,19}$. Concomitante a isso houve aumento da inatividade física causada por mecanização e tecnificação de muitas tarefas cotidianas, aumento do número de veículos por habitantes e maior tempo diante da televisão, jogos e internet ${ }^{20-21}$.

Nos Estados Unidos, o câncer também é a segunda causa de morte, responsável por $22,9 \%$ de todas as mortes no ano de 2001. O pico de sua incidência ocorreu em meados dos anos 90, com pequena queda a partir de 1996. Os principais tipos de câncer na estimativa de mortalidade foram, em 2004, pulmão (32\% para homens e $25 \%$ para mulheres), próstata (10\%), mama $(15 \%)$ e cólon e reto $(10 \%)^{22}$. De modo geral, os países desenvolvidos apresentam coeficientes de mortalidade por neoplasia mais elevados do que países em desenvolvimento. A exemplo disso, pode-se comparar os coeficientes padronizados, por 100 mil habitantes, para a população mundial, no ano de 2000, dos Estados Unidos (161,8 - homens; 116,4 - mulheres), Canadá $(160,5 ; 116,7)$, França $(201,5 ; 98,0)$ e Alemanha $(176,6 ; 116,9)$, com o Chile $(141,2$; $108,7)$, Colômbia $(116,1 ; 106,5)$, Cuba $(141,0 ; 104,0)$ e México $(102,5 ; 106,3)^{23}$. Apesar de maiores, os coeficientes nos países desenvolvidos apresentam tendência decrescente nos últimos anos. Levi et al. ${ }^{24} \mathrm{en}$ contraram, nos países da União Européia, uma redução de 7,2\% em todos os tipos de câncer entre 1988 e 1996. Os principais motivos que levaram ao declínio dos mais prevalentes tipos de câncer foram o aumento no screening precoce e na qualidade dos exames para diagnóstico dos mes- mos, bem como a melhoria dos tratamen$\operatorname{tos}^{24}$.

No período estudado, os homens apresentaram maiores coeficientes de mortalidade por neoplasia quando comparados às mulheres. Segundo dados analisados por Kligerman ${ }^{25}$, quando as neoplasias malignas são analisadas segundo o sexo, observa-se que as maiores incidências ocorrem entre os homens para câncer de pele (não melanoma), próstata, pulmão e estômago, e entre as mulheres para câncer de mama, pele (não melanoma), colo do útero e cólon e reto. As relações numéricas entre o tipo específico de câncer e o seu coeficiente de mortalidade, do ano de 1998, demonstram o motivo da diferença por sexo: para os cânceres de mama e do colo uterino, as proporções entre o número de casos novos e o de óbitos estimados foram, respectivamente, 4,5 e 3,1. Para os homens, tanto para o câncer de estômago (que incide e mata 1,9 vezes mais os homens) como para o de pulmão (que incide 3,0 vezes e mata 2,8 vezes mais também entre eles), a relação entre o número de casos novos e de mortes foi de 1,5, ou seja, os homens brasileiros são acometidos por tumores mais letais ${ }^{26}$. Dados da American Cancer Society ${ }^{22}$ indicam que, nos Estados Unidos, há a mesma tendência de maiores coeficientes para o sexo masculino, com risco de desenvolver câncer de $1 \mathrm{a}$ cada 2 homens e de 1 em cada 3 mulheres. Além disso, as curvas temporais de mortalidade por neoplasia, segundo sexo, são semelhantes às encontradas no presente estudo (Figura 1).

Em relação às diferenças encontradas nos coeficientes para os grupos etários estudados, observaram-se maiores valores para os indivíduos com mais de 60 anos. Estas diferenças também foram evidenciadas na análise dos coeficientes para as capitais, onde os mesmos apresentaram-se crescentes e com aumento maior, ao longo da serie temporal, para a população idosa, comparada a de 30 a 59 anos. De todos os casos de neoplasias, apenas 5 a $10 \%$ são de causa hereditária (predisposição individual), en- 
quanto $80 \%$ a $90 \%$ estão associados a fatores ambientais, ou seja, o cigarro e atividades ocupacionais (câncer de pulmão); a exposição excessiva ao sol (câncer de pele); o consumo de álcool (câncer de estômago); consumo excessivo de gordura e baixo em fibras (câncer de cólon e reto) ${ }^{23,27}$. Segundo a American Cancer Society ${ }^{23}, 77 \%$ de todos os cânceres são diagnosticados em indivíduos com 55 anos ou mais. O envelhecimento, por si só, é fator de risco para as neoplasias, pois deixa os indivíduos mais susceptíveis às transformações malignas. Isso, somado ao fato de as células das pessoas idosas serem expostas por mais tempo aos diferentes fatores de risco para câncer, incluindo a presença de enfermidades crônico-degenerativas, explica, em parte, o porquê de o câncer ser mais freqüente nesses indivíduos ${ }^{27-28}$. Por isso, é importante considerar o conceito de período de latência, ou seja, o período de tempo compreendido entre a exposição aos fatores de risco e o surgimento da doença, visto que no câncer as primeiras manifestações podem surgir após muitos anos de exposição aos fatores de risco, tornando-o uma doença que acomete predominantemente as pessoas de idade mais avançada ${ }^{13}$. Além disso, os avanços nos tratamentos médicos e diagnósticos precoce ao longo do tempo aumentaram a sobrevida dos pacientes com câncer ${ }^{15}$, o que também poderia explicar as diferenças observadas entre os coeficientes de mortalidade nas duas faixas etárias analisadas.

Inquéritos populacionais sobre fatores de risco são raros e esparsos e os estudos epidemiológicos de cunho etiológico em câncer são muito recentes no Brasil $^{15}$. O Inquérito Domiciliar sobre Comportamentos de Risco e Morbidade Referida de Doenças e Agravos Não Transmissíveis $^{29}$, realizado em 2002 e 2003 em 15 capitais brasileiras e no Distrito Federal, mostrou que houve uma elevada prevalência dos principais fatores de risco para o desenvolvimento de doenças neoplásicas. A prevalência de tabagismo variou de 13 a $25 \%$ nas cida- des estudadas, sendo que os maiores percentuais foram observados nas capitais da região Sul. O percentual de indivíduos que referiram consumir frutas, verduras e legumes cinco vezes ou mais por semana variou entre 51 a $81 \%$ e os menores valores foram observados nas duas capitais da região Norte. A prevalência de sobrepeso e obesidade também foi elevada nos locais estudados, chegando a $46,4 \%$ no Rio de Janeiro. Em relação ao consumo atual de bebidas alcoólicas, Florianópolis teve o maior percentual $58,6 \%$. A prevalência de inatividade física também foi elevada, variando de 28 a $55 \%$ nas cidades estudadas. Apesar das elevadas prevalências de fatores de risco, houve um declínio dos coeficientes de mortalidade de alguns tipos de câncer, como o de estômago e de pulmão, possivelmente devido à diminuição da ingestão de nitritos e nitratos, aumento no consumo de vegetais e frutas frescas e o controle do tabagismo, respectivamente ${ }^{15}$. Entretanto, o inquérito mostrou que os cânceres de pulmão e de mama são mais freqüentes nas regiões Sul e Sudeste, enquanto os cânceres de estômago e de colo do útero têm maior ocorrência nas regiões Norte e Nordeste, conforme a prevalência dos seus fatores de risco nestas regiões ${ }^{29}$.

No presente estudo as capitais das regiões Sul e Sudeste apresentaram os maiores coeficientes de mortalidade por neoplasia quando comparadas às capitais das regiões Norte, Nordeste e CentroOeste, que apresentaram coeficientes menores, porém incrementos maiores ao longo da serie temporal (Figuras 2 a 5). Wünsch Filho e Moncau ${ }^{15}$ analisaram a mortalidade por câncer no Brasil de 1980 a 1995 e observaram que o risco de morte por câncer foi maior nas regiões Sul e Sudeste, mas com coeficientes de mortalidade decrescendo nestas regiões, as mais desenvolvidas do país, enquanto as regiões Norte, Nordeste e Centro-Oeste, menos desenvolvidas, apresentaram coeficientes mais baixos, porém com tendên- 
cia ascendente. As regiões geográficas do Brasil, devido às suas heterogeneidades culturais, demográficas, socioeconômicas (grau de industrialização e urbanização) e políticas, têm suas populações submetidas a fatores de risco diferentes. Também são diferentes nas diversas regiões o acesso ao diagnóstico e tratamento do câncer, as qualidades da assistência prestada, a capacidade diagnóstica e a qualidade das informações fornecidas ${ }^{30,25}$. A maior qualidade na prevenção primária e secundária, bem como a melhoria na sobrevida de pacientes com diagnóstico de câncer podem ter contribuído para o menor aumento da mortalidade observadas nas regiões Sul e Sudeste, as mais desenvolvidas do país. Já a redução das mortes por causas mal definidas, por exemplo, pode ser fator contribuinte para o incremento dos coeficientes de mortalidade por neoplasia, observado nas capitais das regiões Norte, Nordeste e CentroOeste, ao mesmo tempo em que a menor competência na compilação dos dados pode ser responsável pelos menores coeficientes encontrados nessas capitais. Em 1990, a proporção de mortes por causas mal definidas foi de 25,37\% em Manaus, $2,93 \%$ em Recife e $16,12 \%$ em Cuiabá, enquanto em 2000 foi de 24,02\%, 1,16\% e $12,60 \%$, respectivamente ${ }^{31}$. Embora a proporção de mortes por causas mal definidas tenha diminuído, ela ainda continua elevada, indicando que os serviços de diagnóstico ou assistência à saúde são inadequados nesses locais.

Este estudo mostrou que a mortalidade por doenças neoplásicas apresentou tendência crescente no período de 1980 a 2000, em ambos os sexos e na maioria das capitais investigadas, estando de acordo com alguns estudos que sugerem que, com o passar dos anos, o câncer poderá superar a mortalidade por doenças cardiovasculares ${ }^{15}$. Comparando o comportamento do câncer com o de outras doenças, Mansur et al. ${ }^{32}$ verificaram uma tendência decrescente do risco de morte por doenças cardiovasculares, em ho- mens e mulheres da população brasileira, entre 1979 a 1996.

Estudos de tendência de mortalidade são úteis não somente para avaliar o problema do câncer, mas também para estimar a efetividade das estratégias primárias e secundárias no controle das doenças neoplásicas, e ainda para avaliar a qualidade do impacto do tratamento na sobrevida dos indivíduos doentes ${ }^{15}$. Embora outras comparações sejam possíveis, as feitas no presente estudo demonstraram a importância de se dispor, regionalmente, por sexo e idade, dos coeficientes de mortalidade por neoplasia para um planejamento adequado em termos da priorização, implantação e avaliação de programas de controle e de serviços assistenciais.

\section{Conclusões}

Os coeficientes de mortalidade por neoplasia apresentaram-se maiores para o sexo masculino, bem como para a faixa etária acima de 60 anos, provavelmente, devido a maior exposição aos fatores de risco, como fumo, álcool, hábitos alimentares e nutrição, atividades ocupacionais, sedentarismo e obesidade. As diferenças entre os coeficientes segundo sexo são influenciadas pela maior prevalência de câncer de maior letalidade nos homens. As regiões mais desenvolvidas do país, Sul e Sudeste, seguem tendência crescente, com coeficientes elevados de mortalidade, porém com menor aumento ao longo da série temporal. Esse menor aumento da mortalidade é caracterizado por maior avanço no processo de transição demográfica e epidemiológica, e melhores serviços de assistência médica. Ainda são necessárias melhorias no sistema de informação a fim de se obter dados mais confiáveis em todas as regiões do país, para o adequado planejamento das ações de saúde pública, principalmente nas regiões menos desenvolvidas. Outros estudos são necessários para acompanhar a tendência da mortalidade por câncer no Brasil. 
1. Kac G, Velásquez-Melendéz G. A transição nutricional e epidemiologia da obesidade na América Latina. Cad Saúde Pública 2003; 19(Suppl 1): S4-S5.

2. Telarolli Junior R, Machado JCMS, Carvalho F. Perfil demográfico e condições sanitárias dos idosos em área urbana do Sudeste do Brasil. Rev Saúde Pública 1996; 30(5): 485-98.

3. Carvalho JAM, Garcia RA. O envelhecimento da população brasileira: um enfoque demográfico. Cad Saúde Pública 2003; 19(3): 725-33.

4. Fundação Instituto Brasileiro de Geografia e Estatística (IBGE). Diretoria de Pesquisas, Censos Demográficos. [publicação on line] Brasília: IBGE; 2000. Disponível em URL: http://www.ibge.gov.br [acessado em (23/11/2003)]

5. Silva Junior JB, Gomes FBC, Cezário AC, Moura L. Doenças não transmissíveis: bases epidemiológicas. In: Rouquayrol MZ, Almeida Filho N. Epidemiologia e saúde. 6a ed. São Paulo: Medsi; 2003.

6. Azevedo G, Mendonça S. Câncer na população feminina brasileira. Rev Saúde Pública 1993; 27(1): 68-75.

7. Buss PM. Promoção da saúde e qualidade de vida. Ciência \& Saúde Coletiva 2000; 5(1): 163-77.

8. Monteiro CA, Mondini L, Costa RBL. Mudanças na composição e adequação nutricional da dieta familiar nas áreas metropolitanas do Brasil (1988-1996). Rev Saúde Pública 2000; 34(3): 251-8.

9. Consenso Latino Americano para Tratamento da Obesidade. [publicação on line] 2000. Disponível URL: www.saudegratuita.com.br/artigos/artigossaude.asp [acesso em 15/09/2003]

10. Sichieri R, Everhart JE, Mendonça GAS. Diet and mortality from common cancers in Brazil: an ecological study. Cad Saúde Pública 1996; 12(1): 53-9.

11. Slatter ML, Curtin KP, Edwards SL, Schaffer DM. Plant foods, fiber, and rectal cancer. Am J Clin Nutr 2004; 79: 274-81.

12. Ministério da Saúde. Fundação Nacional de Saúde. Mortalidade 1998. [publicação on line] 1998. Disponível em URL: www. funasa.gov/sis/pdfs/ mortalidade_1998.pdf [acesso em 06/10/2003]

13. Ministério da Saúde. Instituto Nacional do Câncer. Coordenação Nacional de Controle de Câncer. PROONCO. O problema do câncer no Brasil. [publicação on line] 4 ed. Rio de Janeiro; 1997. Disponível em URL: http:/ /www.inca.gov.br [acesso em 23/11/2003]

14. Faria MAM, Almeida JWR, Zanetta DM. Mortalidade por câncer urbano-industrial da Baixada Santista, SP (Brasil). Rev Saúde Pública 1999; 33(3): 255-61.
15. Wünsch Filho V, Moncau JE. Mortalidade por câncer no Brasil 1980-1995: padrões regionais e tendências temporais. Rev Assoc Med Bras 2002; 48(3): 250-7.

16. Laurenti R, Jorge MHPM, Lebrão ML, Gotlieb SLD. Estatísticas de Saúde. 2a ed. São Paulo: EPU; 1987.

17. Taddei CFG, Ramos LR, Moraes JC, Wajngarten M, Libberman A, Santos SC, et al. Estudo multicêntrico de idosos atendidos em ambulatórios de cardiologia e geriatria de instituições brasileiras. Arq Bras Cardiol 1997; 69(5): 327-33.

18. Zaslavsky C, Gus I. Idoso. Doença cardíaca e comorbidades. Arq Bras Cardiol 2002; 79(6): 635-9.

19. Chopra M, Galbraith S, Darnton-Hill I. A global response to a global problem: the epidemic of overnutrition. Bulletin of the World Health Organization 2002; 80(12): 952-8.

20. Kain J, Vio F, Albala C. Obesity trends and determinant factors in Latin American. Cad Saúde Pública 2003; 19(Suppl 1): S77-S86.

21. Gutiérrez-Fisac JL, Regidor E, García EL, Banegas JRB, Artalejo FR. La epidemia de obesidad y sus factores relacionados: el caso de España. Cad Saúde Pública. 2003; 19 (Supp 1): S101-S10.

22. American Cancer Society. Cancer Statistics: $A$ presentation from the American Cancer Society. Atlanta; 2004.

23. American Cancer Society. Cancer facts and figures 2003. Atlanta; 2003

24. Levi F, Lucchini F, Negri E, Vecchia CL. The decline in cancer mortality in the European Union, 1988-1996. Eur J Cancer 2000; 36: 1965-8.

25. Kligerman J. Estimativas sobre a incidência e mortalidade por câncer no Brasil - 2002. Rev Bras Cancerologia 2002; 48(2): 175-9.

26. Moraes MF. Estimativa da incidência e mortalidade por câncer no Brasil em 1998. Rev Bras Cancerologia [periódico on line] 1998; 44(1). Disponível em URL: http:/ /www.inca.gov.br/rbc [acesso em 24/06/2004]

27. Ministério da Saúde. Instituto Nacional do Câncer. Coordenação Nacional de Controle de Tabagismo. CONTAPP. Falando sobre câncer e seus fatores de risco. [publicação on line] Rio de Janeiro; 1996. Disponível em URL: http://www.inca.gov.br/cancer/o que causa o câncer [acesso em 23/06/2004]

28. Mazza A, Casiglia E, Scarpa, R, Tikhonoff V, Pizziol A, Sica E, et al. Predictors of cancer mortality in elderly subjects. Eur J Epidemiol 1999; 15: 421-27. 
29. Ministério da Saúde. Instituto Nacional do Câncer. Inquérito Domiciliar sobre Comportamentos de Risco e Morbidade Referida de Doenças e Agravos não Transmissíveis. Brasil, 15 capitais e Distrito Federal 20022003. [publicação on line]. Disponível em URL: http:// www.inca.gov.br/inquerito [acesso em 05/08/200)]

30. Kligerman J. Estimativas sobre a incidência e mortalidade por câncer no Brasil - 2000. Rev Bras Cancerologia [periódico on line] 2000; 46(2). Disponível em URL: http:/ /www.inca.gov.br/rbc [acesso em 24/06/2004]
31. Ministério da Saúde. Fundação Nacional da Saúde. DATASUS. Sistema de Informação sobre Mortalidade: dados de declaração de óbito. [publicação on line] Brasília: MS; 1990 e 2000. Disponível em URL: http:// www.saude.gov.br/datasus [acesso em 23/11/2003]

32. Mansur AP, Favarato D, Souza MFM, Avakian SD, Aldrighi JM, Cézar LAM, et al. Tendência do risco de morte por doenças circulatórias no Brasil de 1979 a 1996. Arq Bras Cardiol. 2001; 77(6):497-503.

Recebido em: 18/04/05 Versão reformulada reapresentada em: 07/09/05 Aprovado em: 08/09/05 